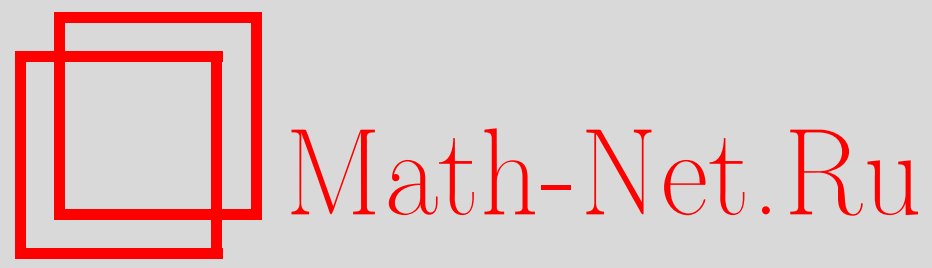

Ю. А. Неретин, О разделении спектров в анализе ядер Березина, Функи. анализ и его прил., 2000, том 34, выпуск 3, 49-62

DOI: https://doi.org/10.4213/faa311

Использование Общероссийского математического портала MathNet.Ru подразумевает, что вы прочитали и согласны с пользовательским соглашением

http://www . mathnet.ru/rus/agreement

Параметры загрузки:

IP: 18.234 .156 .22

26 апреля 2023 г., 18:18:46

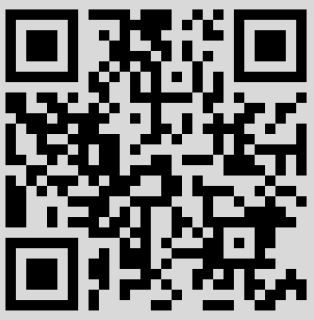


Функциональньй анализ и его приложения

2000, т. 34, вып. 3, с. 49-62

УДК 512.81

\title{
О разделении спектров в анализе ядер Березина*
}

\author{
(C) 2000. Ю. А. НЕРетин
}

Пусть $G$ - классическая вещественная группа Ли, а $K$ - ее максимальная компактная подгруппа. Существует каноническое вложение симметрического пространства $G / K$ в некоторое некомпактное эрмитово симметрическое пространство $G^{*} / K^{*}$, такое, что $\operatorname{dim}_{\mathbb{R}} G / K=\operatorname{dim}_{\mathbb{C}} G^{*} / K^{*}$ (список таких вложений симметрических пространств содержится, например, в [16, добавление A]). Рассмотрим задачу об ограничении представления группы $G^{*}$ со старшим весом на подгруппу $G$. Мы используем для подобных ограничений термин «кернпредставления» (по аналогии с керн-функцией).

Первая серьезная работа о керн-представлениях была опубликована Березиным [2] в 1978 г. незадолго до гибели (доказательства были опубликованы лишь в 1994 г. в [25]). В последние несколько лет интерес к этому объекту возобновился, отчасти в связи с продолжением работы Березина, отчасти по независимым причинам $[25,20,21,23,3,17,4,19]$. Оказалось, в частности, что спектры керн-представлений весьма замысловаты (например они строго включают все, что может встретиться в спектрах двойственных пар Хау; см. [18]). В [20] керн-представления были использованы для построения экзотических унитарных представлений групп $\mathrm{O}(p, q), \mathrm{U}(p, q), \operatorname{Sp}(p, q)$.

Отметим, что в случае пространств скалярнозначных функций (см. §1) предел керн-представлений, когда параметр представления $\alpha$ стремится к $+\infty$, есть обычное представление группы $G$ в $L^{2}(G / K)$, т.е. керн-представления можно рассматривать как естественную деформацию пространств $L^{2}(G / K)$ (в векторнозначном случае деформируется пространство сечений соответствующего векторного расслоения на $G / K)$.

Цель настоящей статьи - разложение керн-представлений на «блоки» с более или менее однотипными спектрами (фактически мы рассматриваем лишь случай $G=\mathrm{O}(p, q)$, группы $G=\mathrm{U}(p, q), \operatorname{Sp}(p, q)$ разбираются аналогично с помощью интегралов $(2.5),(2.6))$. Идея построения таких разложений в различных задачах гармонического анализа является довольно старой (см. [8]). В [22] были введены некоммутативные пространства Харди, позволяющие отделить один из таких блоков - голоморфные дискретные серии (эта работа породила довольно обширную литературу; см. обзор [6]). В недавних работах $[9,14]$ было получено разделение спектров для гиперболоидов. Наш метод основан на теоремах об ограничении разрывных функций на подмногообразия (в $[15,20,3,17]$ этим способом проводилось выделение дискретной части спектра).

В $\S 1$ мы определяем керн-представления группы $\mathrm{O}(p, q)$ (в пространствах скалярнозначных функций). В $\S 2$ вычисляются некоторые интегралы по ортогональной группе, которые кажутся любопытными и сами по себе. В $\S 3$

* Исследования поддержаны грантом РФФИ 98-01-00303 и Российской программой поддержки ведущих научных школ (грант 96-01-96249). 
мы доказываем существование операторов ограничения. В $\S 4$ мы устанавливаем аналогичные утверждения для общих керн-представлений (в пространствах векторнозначных функций).

Я благодарю Г. И. Ольшанского, В. Ф. Молчанова и Б. Орстеда за обсуждение этого предмета.

\section{$\S 1$. Керн-представления}

1.0. Положительно определенные ядра. Напомним, что положительно определенным ядром на множестве $\mathscr{X}$ называется функция $K(x, y)$ на $\mathscr{X} \times \mathscr{X}$, такая, что для любых $x_{1}, \ldots, x_{n} \in \mathscr{X}$ матрица

$$
\left(\begin{array}{ccc}
K\left(x_{1}, x_{1}\right) & \ldots & K\left(x_{1}, x_{n}\right) \\
\vdots & \ddots & \vdots \\
K\left(x_{n}, x_{1}\right) & \ldots & K\left(x_{n}, x_{n}\right)
\end{array}\right)
$$

неотрицательно определена. Положительно определенное ядро определяет однозначно (с точностью до естественной эквивалентности) гильбертово пространство $H$ и систему векторов $\theta_{x}$ (переполненный базис), нумеруемую точками $x \in \mathscr{X}$, такие, что

1) $\left\langle\theta_{x}, \theta_{y}\right\rangle_{H}=K(x, y)$ для всех $y, x \in \mathscr{X}$;

2) линейная оболочка векторов $\theta_{x}$ плотна в $H$.

Далее каждому вектору $v \in H$ ставится в соответствие функция $f_{v}(x)$ на $\mathscr{X}$ по правилу

$$
f_{v}(x)=\left\langle v, \theta_{x}\right\rangle_{H} .
$$

Пространство всех таких функций мы обозначим через $H^{\circ}$.

1.1. Матричные шары. Пусть $p \leqslant q$. Через $\mathrm{B}_{p, q}$ мы обозначим пространство вещественных матриц размера $p \times q$ с нормой $<1$ (под нормой матрицы подразумевается норма оператора в евклидовом пространстве), а через $\overline{\mathrm{B}}_{p, q}$ замыкание множества $\mathrm{B}_{p, q}$ в $\mathbb{R}^{p q}$, т. е. множество матриц с нормой $\leqslant 1$.

Обозначим через $\mathrm{O}(p, q)$ группу вещественных матриц $g=\left(\begin{array}{ll}a & b \\ c & d\end{array}\right)$ размера $(p+q) \times(p+q)$, сохраняющих симметрическую билинейную форму с матрицей $\left(\begin{array}{cc}1 & 0 \\ 0 & -1\end{array}\right)$. Группа $\mathrm{O}(p, q)$ действует на матричном шаре $\mathrm{B}_{p, q}$ (а также на $\overline{\mathrm{B}}_{p, q}$ ) дробно-линейными преобразованиями вида

$$
z \mapsto z^{[g]}:=(a+z c)^{-1}(b+z d) .
$$

Легко проверяется, что это действие транзитивно на $\mathrm{B}_{p, q}$, а стабилизатор точки $z=0$ состоит из матриц вида $\left(\begin{array}{ll}a & 0 \\ 0 & d\end{array}\right)$, где $a \in \mathrm{O}(p), d \in \mathrm{O}(q)$. Поэтому

$$
\mathrm{B}_{p, q} \simeq \mathrm{O}(p, q) / \mathrm{O}(p) \times \mathrm{O}(q) .
$$

Через $\mathrm{M}_{h}$ мы обозначим множество матриц $z \in \overline{\mathrm{B}}_{p, q}$, таких, что ранг матрицы $1-z z^{t}$ равен $h$. Легко видеть, что множества $\mathrm{M}_{h}$ - это в точности орбиты группы $\mathrm{O}(p, q)$ на границе замкнутого матричного шара $\overline{\mathrm{B}}_{p, q}$. Пусть 
$\overline{\mathrm{M}}_{h}$ обозначает замыкание орбиты $\mathrm{M}_{h}$ :

$$
\overline{\mathrm{M}}_{h}=\bigcup_{r \leqslant h} \mathrm{M}_{r} .
$$

1.2. Пространства Березина. Пусть $\alpha=0,1, \ldots, p-1$ или $\alpha>p-1$. Ядро Березина $K_{\alpha}=K_{\alpha}(z, u)$ - это функция на $\mathrm{B}_{p, q} \times \mathrm{B}_{p, q}$, заданная формулой

$$
K_{\alpha}(z, u)=\operatorname{det}\left(1-z u^{t}\right)^{-\alpha}=\operatorname{det}\left(1-u z^{t}\right)^{-\alpha} .
$$

Согласно известной теореме Березина [1], при указанных значениях параметра $\alpha$ это ядро положительно определено и поэтому определяет некоторое гильбертово пространство. Сейчас мы это пространство опишем.

Обозначим через $\mathscr{D}^{\prime}\left(\mathrm{B}_{p, q}\right)$ пространство обобщенных функций с носителем, содержащимся в открытом матричном шаре $\mathrm{B}_{p, q}$. Введем в $\mathscr{D}^{\prime}\left(\mathrm{B}_{p, q}\right)$ скалярное произведение по формуле

$$
\langle\phi, \psi\rangle_{\alpha}:=\left\{\phi(z) \psi(u), \operatorname{det}\left(1-z u^{t}\right)^{-\alpha}\right\} .
$$

Пространство Березина $H_{\alpha}=H_{\alpha}\left(\mathrm{B}_{p, q}\right)$ есть пополнение предгильбертова пространства $\mathscr{D}^{\prime}\left(\mathrm{B}_{p, q}\right)$ по этому скалярному произведению (переполненный базис $\theta_{x}$ состоит из $\delta$-функций).

1.3. Двойственная реализация пространств Березина и керн-представления. Обозначим через $\delta_{a}$ дельта-функцию, сосредоточенную в точке $a \in \mathrm{B}_{p, q}$. Поставим в соответствие каждому элементу $v \in H_{\alpha}\left(\mathrm{B}_{p, q}\right)$ функцию $f_{v}$ на $\mathrm{B}_{p, q}$, заданную формулой

$$
f_{v}(z)=\left\langle v, \delta_{z}\right\rangle_{H_{\alpha}} .
$$

Таким образом мы получили некоторое пространство $H_{\alpha}^{\circ}\left(\mathrm{B}_{p, q}\right) \simeq H_{\alpha}\left(\mathrm{B}_{p, q}\right)$, состоящее уже из настоящих функций на $\mathrm{B}_{p, q}$.

Легко проверяется, что элементы пространства $H_{\alpha}^{\circ}\left(\mathrm{B}_{p, q}\right)$ являются вещественно-аналитическими функциями на $\mathrm{B}_{p, q}$. Далее мы определим унитарное представление $T_{\alpha}$ группы $\mathrm{O}(p, q)$ в $H_{\alpha}^{\circ}\left(\mathrm{B}_{p, q}\right)$ по формуле

$$
T_{\alpha}\left(\begin{array}{ll}
a & b \\
c & d
\end{array}\right) f(z)=\operatorname{det}(a+z c)^{-\alpha} f\left((a+z c)^{-1}(b+z d)\right) .
$$

Представления $T_{\alpha}$ мы будем называть керн-представлениями.

ЗАмечАниЕ. Березин [2] в случае эрмитовых симметрических пространств использовал термин «каноническое представление», заимствовав его из [26]. Этот термин может иметь разный смысл; фактически в работе Вершика, Гельфанда и Граева [26] и последующих работах тех же авторов термин «каноническое представление» используется для конструкций типа мультипликативного интеграла и для безгранично делимых представлений.

1.4. Голоморфное продолжение. Обозначим через $\mathrm{B}_{p, q}(\mathbb{C})$ множество всех комплексных матриц размера $p \times q$ с нормой $<1$. Ядро $K_{\alpha}$ продолжается до функции $\operatorname{det}\left(1-z u^{*}\right)^{-\alpha}$ на $\mathrm{B}_{p, q}(\mathbb{C}) \times \mathrm{B}_{p, q}(\mathbb{C})$, голоморфной по аргументу $z$ и антиголоморфной по аргументу $u$, причем она остается положительно определенной [1]. Отсюда легко выводится, что функции $f \in H_{\alpha}^{\circ}\left(\mathrm{B}_{p, q}\right)$ продолжаются до голоморфных функций в $\mathrm{B}_{p, q}(\mathbb{C})$. 


\section{§2. Некоторые интегралы по ортогональной группе}

Цель этого параграфа - вычисление интеграла (2.3), который в дальнейшем используется в доказательстве теоремы 3.2.

Через $[A]_{p}$ мы будем обозначать левый верхний угол матрицы $A$ размера $p \times p$. Меру Хаара на группе $\mathrm{SO}(n)$ мы будем обозначать через $d \sigma_{n}(g)$.

2.1. Отображения $\Upsilon^{m}$. Запишем матрицу $g \in \mathrm{SO}(n)$ в виде блочной матрицы $\left(\begin{array}{cc}P & Q \\ R & T\end{array}\right)$ размера $(m+(n-m)) \times(m+(n-m))$. Рассмотрим определенное почти всюду отображение

$$
\Upsilon^{m}:\left(\begin{array}{cc}
P & Q \\
R & T
\end{array}\right) \longmapsto T-R(1+P)^{-1} Q .
$$

ПРЕДЛОЖЕНИЕ 2.1. (а) $\Upsilon^{m}$ есть отображение группь $\mathrm{SO}(n)$ в $\mathrm{SO}(n-m)$; (b) $\Upsilon^{k} \circ \Upsilon^{m}=\Upsilon^{k+m}$;

(c) если $S=(g-1) /(g+1)$, то $\{S\}_{p}=\left(\Upsilon^{n-p}(g)-1\right) /\left(\Upsilon^{n-p}(g)+1\right)$, где через $\{S\}_{p}$ обозначен правый нижний угол матрищь $S$ размера $p \times p$.

ЗАмечАниЕ. Отображение $\Upsilon$ является значением характеристической функции Лифшица $\chi(z)=T+z R(1-z P)^{-1} Q$ при $z=-1$ (см. [13]).

ДОКАЗАТЕЛЬСТВО ПРЕДЛОЖЕНИЯ 2.1. Утверждение (с) проверяется прямым вычислением с помощью следующей формулы Фробениуса $[7, \S$ II.4] для обращения блочной матрицы:

$$
\left(\begin{array}{ll}
A & B \\
C & D
\end{array}\right)^{-1}=\left(\begin{array}{cc}
A^{-1}+A^{-1} B\left(D-C A^{-1} B\right)^{-1} C A^{-1} & -A^{-1} B\left(D-C A^{-1} B\right)^{-1} \\
-\left(D-C A^{-1} B\right)^{-1} C A^{-1} & \left(D-C A^{-1} B\right)^{-1}
\end{array}\right) .
$$

Утверждения (a), (b) следуют из (c).

ПреДЛОЖЕНИЕ 2.2. Пусть $A, B \in \mathrm{SO}(n-m)$. Тогда

$$
\Upsilon^{m}\left[\left(\begin{array}{ll}
1 & 0 \\
0 & A
\end{array}\right) g\left(\begin{array}{cc}
1 & 0 \\
0 & B
\end{array}\right)\right]=A \Upsilon^{m}(g) B
$$

ДоКАЗАТЕЛЬСТВо очевидно.

Объясним теперь смысл отображения $\Upsilon$. Обозначим через Mat $_{n}$ пространство матриц размера $n \times n$. Обобщенно дробно-линейными отображениями $\mathrm{Mat}_{n} \rightarrow \mathrm{Mat}_{k}$ называются (определенные почти всюду) отображения вида

$$
Z \mapsto K+L Z(1-N Z)^{-1} M,
$$

где $N$ - матрица размера $n \times n, K$ - матрица размера $k \times k, L$ имеет размеры $k \times n$, а $N$ - размеры $n \times k$. Обычные дробно-линейные отображения $Z \mapsto(A+Z C)^{-1}(B+Z D)$ из $M_{a}$ в себя имеют вид $(2.1)$, но обратное неверно. Более важное для нас обстоятельство - то, что отображения вида $(2.1)$ могут связывать разные матричные пространства (такие отображения вполне естественны с геометрической точки зрения; см. [16, §VI.3]). Отображение $\Upsilon^{m}: \mathrm{SO}(n) \mapsto \mathrm{SO}(n-m)$ имеет вид $(2.1)$, а именно $K=0$, матрица $N$ - блочная матрица $\left(\begin{array}{cc}-1 & 0 \\ 0 & 0\end{array}\right)$ размера $(m+(n-m)) \times(m+(n-m)), L-$ блочная матрица $\left(\begin{array}{ll}0 & 1\end{array}\right)$ размера $(n-m) \times(m+(n-m))$, а $M=L^{t}$. 
Отображение $\Upsilon^{m}$ — это единственное обобщенно дробно-линейное отображение $\Xi: \mathrm{SO}(n) \rightarrow \mathrm{SO}(n-m)$, удовлетворяющее двум условиям:

1) $\Xi$ перестановочно с действием группы $\mathrm{SO}(n-m) \times \mathrm{SO}(n-m)$;

2) пусть $h \in \mathrm{SO}(m)$; тогда $\Xi\left[\left(\begin{array}{ll}h & 0 \\ 0 & 1\end{array}\right) g\left(\begin{array}{cc}h^{-1} & 0 \\ 0 & 1\end{array}\right)\right]=\Xi(g)$.

Отметим, что при $m=1$ достаточно лишь первого условия.

2.2. Другое доказательство предложения 2.1. Рассмотрим теперь пространство $V_{n}:=\mathbb{R}^{n} \oplus \mathbb{R}^{n}$, снабженное знаконеопределенной билинейной формой

$$
L_{n}\left((x, y),\left(x^{\prime}, y^{\prime}\right)\right):=\sum_{k=1}^{n} x_{k} x_{k}^{\prime}-\sum_{k=1}^{n} y_{k} y_{k}^{\prime} .
$$

Тогда графики ортогональных операторов $\mathbb{R}^{n} \rightarrow \mathbb{R}^{n}$ становятся максимальными $L_{n}$-изотропными подпространствами в $V_{n}=\mathbb{R}^{n} \oplus \mathbb{R}^{n}$.

Далее, для любого линейного отношения $M: V_{n} \rightrightarrows V_{n-m}$ (т. е. подпространства $M \subset V_{n} \oplus V_{n-m}$; подробнее см. [16]) и любого подпространства $H \subset V_{n}$ стандартным образом определено подпространство $M H \subset V_{n-m}$. Оно состоит из векторов $y \in V_{n-m}$, для которых существует $x \in H$, такой, что $(x, y) \in M$.

Определим линейное отношение $\mathscr{Z}_{n}^{n-m}: V_{n} \rightrightarrows V_{n-m}$, состоящее из векторов вида

$$
(\{(v, h),(w,-h)\},\{v, w\}) \in\left\{\mathbb{R}^{n} \oplus \mathbb{R}^{n}\right\} \oplus\left\{\mathbb{R}^{n-m} \oplus \mathbb{R}^{n-m}\right\} .
$$

Условие $(u, v),\left(u^{\prime}, v^{\prime}\right) \in \mathscr{Z}_{n}^{n-m}$ влечет за собой равенство $L_{n}\left(u, u^{\prime}\right)=L_{n-m}\left(v, v^{\prime}\right)$. Поэтому отображение $H \mapsto \mathscr{Z}_{n}^{n-m} H$ переводит изотропные подпространства в изотропные.

Легко проверить, что отображение $\Upsilon^{m}$ на этом языке в точности совпадает с отображением изотропных грассманианов, заданным формулой $H \mapsto \mathscr{Z}_{n}^{n-m} H$. После этого предложение 2.1 становится очевидным.

ЗАмЕчАниЕ. Обобщенно дробно-линейные отображения, переводящие группу $\mathrm{O}(n)$ в $\mathrm{O}(n-m)$, находятся во взаимно однозначном соответствии с максимальными изотропными подпространствами в $V_{n} \oplus V_{n-m}$.

2.3. Проекция меры Хаара. Обозначим через $I$ отрезок $[-1,1]$. Рассмотрим отображение

$$
\Xi: \mathrm{SO}(n) \rightarrow \mathrm{SO}(n-1) \times I,
$$

заданное формулой (см. обозначение в начале параграфа)

$$
\Xi(g)=\left(\Upsilon^{1}(g),[g]_{1}\right) .
$$

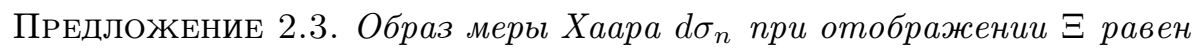

$$
\text { const } \cdot\left(1-x^{2}\right)^{(n-3) / 2} d x d \sigma_{n-1} \text {. }
$$

ДоКАЗАтЕЛЬСтво. В силу $\mathrm{SO}(n-1)$-эквивариантности отображения $\Upsilon^{1}$ (предложение 2.2) образ меры Хаара имеет вид $\phi(x) d x d \sigma_{n-1}$. С другой стороны, проекция меры Хаapa на $I$ равна const $\cdot\left(1-x^{2}\right)^{(n-3) / 2}$, и это дает явный вид функции $\phi(x)$.

2.4. Отображение ортогональной группы на куб. Рассмотрим отображения

$$
\mathrm{SO}(n) \rightarrow \mathrm{SO}(n-1) \times[-1,1] \rightarrow \mathrm{SO}(n-2) \times[-1,1] \times[-1,1] \rightarrow \ldots
$$


В итоге мы получим отображение $\mathrm{SO}(n)$ на множество $[-1,1]^{n-1}$, заданное формулой

$$
\left(x_{1}, x_{2}, \ldots, x_{n-1}\right)=\left(\left[\Upsilon^{n-2}(g)\right]_{1}, \ldots,\left[\Upsilon^{1}(g)\right]_{1},[g]_{1}\right) .
$$

В силу предложения 2.3 образ меры Хаара при этом отображении равен

$$
d \mu\left(x_{1}, x_{2}, \ldots, x_{n-1}\right)=\mathrm{const} \cdot \prod_{j=1}^{n-1}\left(1-x_{j}^{2}\right)^{(j-2) / 2} \prod_{j=1}^{n-1} d x_{j} .
$$

Поэтому для любой функции $f$ от $n-1$ переменных $x_{1}, \ldots, x_{n-1} \in[-1,1]$ выполняется равенство

$$
\begin{aligned}
\int_{\mathrm{SO}(n)} & f\left(\left[\Upsilon^{n-2}(g)\right]_{1}, \ldots,\left[\Upsilon^{1}(g)\right]_{1},[g]_{1}\right) d \sigma_{n}(g) \\
= & \int_{[-1,1]^{n-1}} f\left(x_{1}, x_{2}, \ldots, x_{n-1}\right) d \mu\left(x_{1}, x_{2}, \ldots, x_{n-1}\right) .
\end{aligned}
$$

\section{5. Мультипликативность.}

ПреДЛОЖенИЕ 2.4. Пусть $g \in \mathrm{SO}(n)$. Пусть $m<p \leqslant n$. Тогда

$$
\operatorname{det}\left(1+[g]_{p}\right)=\operatorname{det}\left(1+[g]_{m}\right) \operatorname{det}\left(1+\left[\Upsilon^{m}(g)\right]_{p-m}\right) .
$$

ДоКАЗАТЕЛЬСтво. Запишем $g$ в виде блочной матрицы размера $(m+(p-m)$ $+(n-p)) \times(m+(p-m)+(n-p)):$

$$
g=\left(\begin{array}{ccc}
P & Q_{1} & Q_{2} \\
R_{1} & T_{11} & T_{12} \\
R_{2} & T_{21} & T_{22}
\end{array}\right)
$$

Тогда по обычной формуле для определителя блочной матрицы

$$
\operatorname{det}\left[1+\left(\begin{array}{cc}
P & Q_{1} \\
R_{1} & T_{11}
\end{array}\right)\right]=\operatorname{det}(1+P) \operatorname{det}\left(1+T_{11}-R_{1}(1+P)^{-1} Q_{1}\right) .
$$

С другой стороны,

$$
\Upsilon^{m}(g)=\left(\begin{array}{ll}
T_{11}-R_{1}(1+P)^{-1} Q_{1} & T_{12}-R_{1}(1+P)^{-1} Q_{2} \\
T_{21}-R_{2}(1+P)^{-1} Q_{1} & T_{22}-R_{2}(1+P)^{-1} Q_{2}
\end{array}\right),
$$

и теперь утверждение становится очевидным.

\section{6. Некоторые интегралы.}

Теорема 2.5. Нормируем меру Хаара на $\mathrm{SO}(n)$ так, ито мера всей группь равна 1. Пусть $\lambda_{1}, \ldots, \lambda_{n-1} \in \mathbb{C}, \lambda_{n}=0$, причем $\operatorname{Re} \lambda_{k}>-(n-k) / 2$ для всех $k=0,1, \ldots, n$. Тогда

$$
\int_{\mathrm{SO}(n)} \prod_{k=1}^{n-1} \operatorname{det}\left(1+[g]_{k}\right)^{\lambda_{k}-\lambda_{k+1}} d \sigma_{n}(g)=\prod_{k=1}^{n-1} \frac{\Gamma(n-k) \Gamma\left(\lambda_{k}+(n-k) / 2\right)}{\Gamma((n-k) / 2) \Gamma\left(\lambda_{k}+n-k\right)} .
$$


ДокАЗАТЕЛЬСТво. Применим формулу (2.2) и предложение 2.4. Тогда наш интеграл распадется в произведение интегралов вида

$$
\begin{aligned}
\int_{-1}^{1}\left(1-x^{2}\right)^{(n-k-2) / 2}(1+x)^{\lambda_{k}} d x & =2^{\lambda_{k}+n-3} B\left(\lambda_{k}+\frac{n-k}{2}, \frac{n-k}{2}\right) \\
& =2^{\lambda_{k}+n-k-3} \frac{\Gamma\left(\lambda_{k}+(n-k) / 2\right) \Gamma((n-k) / 2)}{\Gamma\left(\lambda_{k}+n-k\right)} .
\end{aligned}
$$

\section{7. Некоторые интегралы по унитарной и симплектической груп-} пам. Следующие интегралы вычисляются тем же способом, только очевидный интеграл (2.4) заменяется на более замысловатые интегралы по кругу (шару) $|z| \leqslant 1$ в $\mathbb{C}$ или в $\mathbb{H}$ :

$$
\begin{aligned}
& \int_{\mathrm{U}(n)} \prod_{k=1}^{n} \operatorname{det}\left(1+[g]_{k}\right)^{\lambda_{k}-\lambda_{k+1}} \overline{\operatorname{det}\left(1+[g]_{k}\right)} \mu_{k}-\mu_{k+1} d \sigma_{n}(g) \\
& =\prod_{k=1}^{n} \frac{\Gamma(n-k+1) \Gamma\left(n-k+1+\lambda_{k}+\mu_{k}\right)}{\Gamma\left(n-k+1+\lambda_{k}\right) \Gamma\left(n-k+1+\mu_{k}\right)}, \\
& \int_{\mathrm{Sp}(n)} \prod_{k=1}^{n}\left|\operatorname{det}\left(1+[g]_{k}\right)\right|^{\lambda_{k}-\lambda_{k+1}} d \sigma_{n}(g) \\
& =\prod_{k=1}^{n} \frac{\Gamma(2(n-k+1)) \Gamma\left(2(n-k+1)+\lambda_{k}+1\right)}{\Gamma\left(2(n-k+1)+\lambda_{k} / 2\right) \Gamma\left(2(n-k+1)+\lambda_{k} / 2+1\right)}
\end{aligned}
$$

(компактную симплектическую группу $\operatorname{Sp}(n)$ мы реализуем как группу унитарных операторов в $n$-мерном кватернионном пространстве $\mathbb{H}^{n}$; любой кватернионный оператор $A$ может рассматриваться как оператор $A_{\mathbb{R}}: \mathbb{R}^{4 n} \rightarrow \mathbb{R}^{4 n}$; под определителем кватернионного оператора $A$ мы подразумеваем $\left.\sqrt[4]{\operatorname{det} A_{\mathbb{R}}}\right)$.

2.8. Другие способы вычисления интегралов (2.3), (2.5), (2.6). После преобразования Кэли $g=(1-T) /(1+T)$ подынтегральные выражения в $(2.3)$ и $(2.6)$ приводятся к виду $\prod \operatorname{det}\left(1+[T]_{k}\right)^{\nu_{k}}$, а подынтегральное выражение в (2.5) приводится к виду $\prod \operatorname{det}\left(1+[T]_{k}\right)^{\nu_{k}} \operatorname{det}\left(1-[T]_{k}\right)^{\tau_{k}}$. Во всех трех случаях может быть применен метод разделения переменных, описанный в [19]. В случае унитарной группы возможны также два других пути: вычисление по методу Гиндикина, т. е. переход к интегрированию по треугольной подгруппе (см. образцы подобных вычислений в $[5$, Ch. 7]), и применение преобразования Лапласа (интеграл в этом случае имеет вид $L^{2}$-скалярного произведения); образец такого вычисления см. в [5, p. 142].

При $\lambda_{1}=\cdots=\lambda_{n}$ интеграл (2.2) превращается в один из интегралов Хуа Ло Кена [11, гл. 2].

\section{§3. Операторы ограничения на границу}

В этом параграфе мы полагаем $p<q$.

3.1. Операторы ограничения на $\mathbf{M}_{\boldsymbol{r}}$. Обозначим через $\mathscr{H}_{\alpha}^{\circ}$ подпространство в $H_{\alpha}^{\circ}\left(\mathrm{B}_{p, q}\right)$, состоящее из функций, продолжимых по аналитичности в некоторую окрестность замкнутого матричного шара $\overline{\mathrm{B}}_{p, q}$. 
ЛЕмма 3.1. Подпространство $\mathscr{H}_{\alpha}^{\circ}$ плотно в $H_{\alpha}^{\circ}$.

ДоКАЗАТЕЛЬСтво. В пространстве $H_{\alpha}^{\circ}$ существует ортогональный базис, состоящий из однородных многочленов (см. $[20, \S 1])$. Оператор $A_{\varepsilon} f(z)=f((1-\varepsilon) z)$ в этом базисе диагонален и имеет собственные числа $(1-\varepsilon)^{k}$. Поэтому для любого $\varepsilon>0$ оператор $A_{\varepsilon}$ является непрерывным оператором $H_{\alpha}^{\circ} \rightarrow H_{\alpha}^{\circ}$. Очевидно, что образ оператора $A_{\varepsilon}$ плотен в $H_{\alpha}^{\circ}$ и содержится в $\mathscr{H}_{\alpha}^{\circ}$.

Через $L_{\text {loc }}^{1}\left(\mathrm{M}_{r}\right)$ мы обозначим пространство локально интегрируемых функций на (вообще говоря некомпактном) многообразии $\mathrm{M}_{r}$ (см. разд. 1.1). Отметим, что группа $\mathrm{O}(p, q)$ действует в $L_{\text {loc }}^{1}\left(\mathrm{M}_{r}\right)$ по той же формуле $(1.1)$, что и в $H_{\alpha}^{\circ}\left(\mathrm{B}_{p, q}\right)$ (нужно лишь считать, что в (1.1) матрица $z$ лежит в $\left.\mathrm{M}_{r}\right)$.

Через $J_{r}$ мы обозначим оператор ограничения функции $f \in \mathscr{H}_{\alpha}^{\circ}$ на многообразие $\mathrm{M}_{r}$.

Теорема 3.2. Пусть $\alpha<(q-p+2 r) / 2$. Тогда оператор $J_{r}$ продолжается до непрерывного оператора $H_{\alpha}^{\circ} \rightarrow L_{\mathrm{loc}}^{1}\left(\mathrm{M}_{r}\right)$.

ЗАмечАниЕ. За исключением случая $\alpha=0$, когда пространство $H_{\alpha}^{\circ}$ состоит лишь из констант, функции $f \in H_{\alpha}^{\circ}$, вообще говоря, разрывны в точках границы матричного шара. Иными словами, если $a$ - точка границы, то линейный функционал $f \mapsto f(a)$ на $H_{\alpha}^{\circ}$ не определен. Поэтому существование оператора ограничения на подмногообразие не является само собой разумеющимся фактом.

Очевидно, что оператор $J_{r}: H_{\alpha}^{\circ} \rightarrow L_{\text {lос }}^{1}\left(\mathrm{M}_{r}\right)$ является $\mathrm{O}(p, q)$-сплетающим. Следовательно, его ядро ker $J_{r}$ является подпредставлением. Поэтому подпредставлением является и ортогональное дополнение $\left(\operatorname{ker} J_{r}\right)^{\perp}$ к $\operatorname{ker} J_{r}$. Оператор $J_{r}$ осушествляет биекцию между $\left(\operatorname{ker} J_{r}\right)^{\perp}$ и образом im $J_{r}$ оператора $J_{r}$. Значит, мы получили в $H_{\alpha}^{\circ}$ некоторое подпредставление, которое естественным образом реализуется в пространстве функций на $\mathrm{M}_{r}$.

3.2. Доказательство теоремы 3.2. В [20] было доказано одно общее утверждение о граничных значениях голоморфных функций (теоремы $2.1,2.2$ ), которое в нашем случае означает следующее.

Теорема 3.3. Пусть $M$ - некоторое компактное подмножество в граниче матричного шара $\mathrm{B}_{p, q}$. Пусть $\mu$ - некоторая мера с носителем в $M$. Допустим, ито

(i) для почти всех $(z, u) \in M \times M$ в смысле меры $\mu \times \mu$ существует предел при $\varepsilon$, стремящемся $к$ нулю справа,

$$
L(z, u)=\lim _{\varepsilon \rightarrow+0} \operatorname{det}\left(1-(1-\varepsilon) z u^{t}\right)^{-\alpha}
$$

(ii) этот предел является мажорируемьм, т. е. существует функиия $h$, интегрируемая по мере $\mu \times \mu$, такая, что

$$
\operatorname{det}\left(1-(1-\varepsilon) z u^{t}\right)^{-\alpha} \leqslant h(z, u) .
$$

Тогда оператор ограничения функиии $f \in H_{\alpha}^{\circ}$ на множество $M$ является непрерывнылм оператором из $H_{\alpha}^{\circ}$ в $L^{1}(M)$.

Мы хотим применить эту теорему к орбите $\mathrm{M}_{r}$. Существование предела в нашем случае очевидно, и фактически в проверке нуждается лишь сходимость интеграла от $L(z, u)=\operatorname{det}\left(1-z u^{*}\right)^{-\alpha}$. Однако на $\mathrm{M}_{r}$ нет разумной канонической меры. Поэтому надежды явно вычислить интеграл нет. Основной шаг 
приводимого ниже доказательства - лемма 3.5, сводящая оценку сходимости интеграла по $\mathrm{O}(p, q)$-орбите $\mathrm{M}_{r}$ к сходимости вычисленного выше интеграла (2.3) по группе $\mathrm{SO}(q+r)$.

Чтобы формально применить теорему, мы возьмем в качестве $M$ произвольное компактное подмножество в многообразии $\mathrm{M}_{r}$, являющееся замыканием своей внутренности. В качестве $\mu$ возьмем (для определенности) поверхностную меру Лебега на $M$. Нам достаточно проверить, что интеграл

$$
\int_{M} \int_{M} \operatorname{det}\left(1-z u^{t}\right)^{-\alpha} d \mu(z) d \mu(u)
$$

сходится. Отсюда будет следовать условие (i), а условие (ii) тогда будет вытекать из очевидного неравенства [20,3.8]

$$
\operatorname{det}\left(1-c^{2} z u^{t}\right)^{-\alpha} \leqslant 2^{p \alpha} \operatorname{det}\left(1-z u^{t}\right)^{-\alpha} .
$$

Достаточно проверить, что интеграл

$$
\int_{M} \operatorname{det}\left(1-z u^{t}\right)^{-\alpha} d \mu(z)
$$

сходится при фиксированном $u$ и оценивается сверху константой, не зависящей от $u$. Как будет показано в дальнейшем, достаточно проверить сходимость интеграла при каком-нибудь одном $u$. Мы начнем с такой проверки и для этого выберем наиболее удобный для наших целей элемент $u$.

Пусть $u=u^{(0)}$ задается блочной $((p-r)+r) \times((p-r)+(q-p+r))$-матрицей $\left(\begin{array}{cc}-1 & 0 \\ 0 & 0\end{array}\right)$. Тогда интеграл (3.2) переписывается в виде

$$
\int_{M} \operatorname{det}[1+z]_{p-r}^{-\alpha} d \mu(z) \text {. }
$$

ЛЕмма 3.4. Интеграл (3.3) сходится.

ЛЕмма 3.5. Запишем матрицу $g \in \mathrm{SO}(q+r)$ в виде блочной матриць $g=$ $\left(\begin{array}{ll}g_{11} & g_{12} \\ g_{21} & g_{22}\end{array}\right)$ размера $(p+(q+r-p)) \times(q+r)$. Тогда $g_{11} \in \overline{\mathrm{M}}_{r}$. Обратно любая матрица $z \in \overline{\mathrm{M}}_{r}$ встраивается в качестве левого верхнего блока в некоторую матричу $g \in \mathrm{SO}(q+r)$.

Доказательство леммы 3.5 очевидно.

ДокАЗАтЕЛЬСтво лЕммы 3.4. Отображение $\tau: g \mapsto g_{11}$, согласно лемме 3.5, переводит $\mathrm{SO}(q+r)$ в $\overline{\mathrm{M}}_{r}$. Очевидно, что образ меры Хаара на $\mathrm{SO}(q+r)$ при отображении $\tau$ является мерой, эквивалентной поверхностной мере Лебега на $\mathrm{M}_{r}$. Поэтому нам достаточно проверить сходимость интеграла

$$
\int_{\mathrm{SO}(q+r)} \operatorname{det}[1+g]_{p-r}^{-\alpha} d \sigma_{q+r}(g) .
$$

Но этот интеграл нами уже был вычислен (нужно подставить $\lambda_{1}=\cdots=$ $\lambda_{p-r-1}=-\alpha, \lambda_{p-r}=\cdots=0$ в формулу $\left.(2.3)\right)$.

Лемма 3.6. Ядро $\operatorname{det}\left(1-z u^{t}\right)^{-\alpha}$ удовлетворяет тождеству

$$
\operatorname{det}\left(1-z^{[g]}\left(u^{[g]}\right)^{t}\right)^{-\alpha}=\operatorname{det}\left(1-z u^{t}\right)^{-\alpha} \operatorname{det}(a+z c)^{\alpha} \operatorname{det}(a+z u)^{\alpha} .
$$

Доказательство очевидно. 
Рассмотрим какое-нибудь компактное подмножество $L \subset \mathrm{O}(p, q)$, такое, что для любого $v \in M$ существует $g_{v} \in L$, такой, что $v^{\left[g_{v}\right]}=u^{(0)}$. Через $K$ мы обозначим множество всех точек в $\mathrm{M}_{r}$ вида $u^{[g]}$, где $u \in M$, а $g \in L$. Очевидно, что $K$ компактно.

Замена переменной $w=z^{[g]}$ в интеграле (3.2) в силу формулы (3.4) приводит его к виду

$$
\int_{\left.M^{[g u}\right]} \operatorname{det}\left(1-u^{(0)} w^{t}\right)^{-\alpha} H(w, u) d \mu(w),
$$

где через $M^{\left[g_{u}\right]}$ обозначен образ множества $M$ при отображении $g_{u}$, а $H(w, u)$ - некоторая функция (произведение якобиана на дополнительный множитель, возникающий из (3.4)). Легко видеть, что функция $H(w, u)$ ограничена некоторой постоянной $C$. Поэтому интеграл (3.5) оценивается сверху величиной

$$
C \int_{K} \operatorname{det}\left(1-u^{[0]} w^{t}\right)^{-\alpha} d \mu(w),
$$

а эта величина не зависит от $u$ и в силу леммы 3.4 конечна. Теорема доказана.

3.3. Ограничение частных производных на границу. Мы можем ограничивать на многообразия $\mathrm{M}_{r}$ не только функции $f \in H_{\alpha}^{\circ}$, но и их частные производные. Существование оператора ограничения для частной производной $\partial / \partial z_{i j}$ зависит от сходимости интеграла

$$
\int_{M} \int_{M}\left|\frac{\partial}{\partial z_{i j}} \frac{\partial}{\partial u_{i j}} \operatorname{det}\left(1-z u^{t}\right)^{-\alpha}\right| d z d u
$$

где $M \subset \mathrm{M}_{r}$ - произвольное компактное подмножество. Подынтегральное выражение имеет вид $|P(z, u)| \operatorname{det}\left(1-z u^{t}\right)^{-\alpha-2}$, где $P(z, u)$ - многочлен. Поэтому все наши рассуждения применимы и в этом случае. Мы получаем следующую теорему.

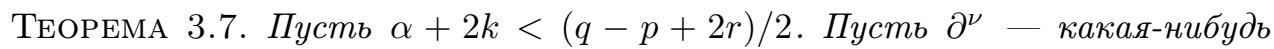
частная производная порядка $k$. Тогда оператор ограничения функции $\partial^{\nu} f(z)$ на $\mathrm{M}_{r}$ является непрерывныл оператором из $H_{\alpha}^{\circ}$ в $L_{\mathrm{loc}}^{1}\left(\mathrm{M}_{r}\right)$.

Сейчас мы слегка формализуем получающуюся картину ${ }^{1}$.

Обозначим через $\mathscr{N}\left(\mathrm{M}_{r}\right)$ конормальное расслоение к $\mathrm{M}_{r}$ в $\mathbb{R}^{p q}$. Через $\mathrm{S}^{k} \mathscr{N}\left(\mathrm{M}_{r}\right)$ мы обозначим $k$-ю симметрическую степень конормального расслоения, а через $\mathscr{F}_{r}^{k}-$ множество всех $f \in \mathscr{H}_{\alpha}^{\circ}$, у которых ограничение всех частных производных порядка $\leqslant k+1$ на $\mathrm{M}_{r}$ равно 0. Тогда элементы факторпространства $\mathscr{F}_{r}^{k-1} / \mathscr{F}_{r}^{k}$ отождествляются с сечениями расслоения $\mathrm{S}^{k} \mathscr{N}\left(\mathrm{M}_{r}\right)$.

Теорема 3.7 тогда влечет за собой следующее высказывание: Пусть $\alpha+2 k<$ $(q-p+2 r) / 2$. Тогда корректно определен непрерьвный «оператор ограничения частных производньх $k$-го порядка» из $H_{\alpha}^{\circ}$ в пространство локально интегрируемьх сечений расслоения $\mathrm{S}^{k} \mathscr{N}\left(\mathrm{M}_{r}\right)$.

3.4. Некоторые комментарии. Итак, нами построен сплетающий оператор $J_{r, k}$ из $H_{\alpha}^{\circ}$ в пространство сечений расслоения $\mathrm{S}^{k} \mathscr{N}\left(\mathrm{M}_{r}\right)$. Образ $\mathrm{im} J_{r, k}$

\footnotetext{
${ }^{1}$ Комбинаторная версия этой конструкции восходит к [12].
} 
оператора $J_{r, k}$ является факторпространством гильбертова пространства и поэтому сам является гильбертовым пространством (отметим, что гильбертова топология в $\mathrm{im} J_{r, k}$ сильнее, чем топология, индуцированная с пространства $\left.L_{\text {loc }}^{1}\right)$. Представление группы $\mathrm{O}(p, q)$ в $\operatorname{im} J_{r, k}$ мы обозначим через

$$
\Xi_{r, k}^{\alpha}=\Xi_{r, k}^{\alpha}(p, q)
$$

Факторпространство произвольного гильбертова пространства $H$ по замкнутому подпространству $L$ канонически отождествляется с ортогональным дополнением $L^{\perp}$ к $L$. Поэтому представление $\Xi_{r, k}^{\alpha}$ может рассматриваться как подпредставление в керн-представлении $T_{\alpha}$.

Итак, мы выделили в пространстве $H_{\alpha}^{\circ}\left(\mathrm{B}_{p, q}\right)$ некоторый естественный набор подпространств (3.6), и эти подпространства мы будем называть блоками.

Если $\alpha$ достаточно велико, а именно $\alpha>(p+q) / 2-1$, то ни один оператор ограничения на $\mathrm{M}_{r}$ не определен, т. е. в этом случае блоки $\Xi_{r, k}^{\alpha}(p, q)$ отсутствуют. Известно, что при указанных ограничениях $(\alpha>(p+q) / 2-1)$ представление группы $\mathrm{O}(p, q)$ в $H_{\alpha}^{\circ}\left(\mathrm{B}_{p, q}\right)$ эквивалентно ее представлению в $L^{2}$ на симметрическом пространстве $\mathrm{B}_{p, q}$ (это утверждение доказано в [21]; ранее такого рода утверждения для керн-представлений разных групп в разной степени общности доказывались в $[2,24,10])$.

При $\alpha<(p+q) / 2-1$ дополнительно к подпредставлению (блоку), эквивалентному $L^{2}\left(\mathrm{~B}_{p, q}\right)$, появляется блок $\Xi_{p-1,0}^{\alpha}$, соответствующий максимальной орбите $\mathrm{M}_{p-1}$ и $k=0$.

При $\alpha<(p+q) / 2-2$ появляется блок $\Xi_{p-2,0}^{\alpha}$.

При $\alpha<(p+q) / 2-3$ появляются два блока: $\Xi_{p-1,1}^{\alpha}$ и $\Xi_{p-3,0}^{\alpha}$, и т.д.

В точке $\alpha=p-1$ (это конец непрерывной серии; см. разд. 1.2) исчезает «самый большой» из блоков, а именно блок, эквивалентный $L^{2}\left(\mathrm{~B}_{p, q}\right)$. В этот же момент исчезают блоки $\Xi_{p-1, k}^{\alpha}$ при $k>0$. Блок $\Xi_{p-1,0}^{\alpha}$ при $\alpha=p-1$ еще остается.

При дальнейшем уменьшении $\alpha$ (проходящем уже дискретно; см. разд. 1.2) продолжают появляться новые блоки, но при этом происходит постепенное исчезновение старых. Наконец при $\alpha=0$ остается лишь блок $\Xi_{0,0}^{0}$, который в данном случае сводится к одномерному представлению.

Теперь мы вкратце обсудим спектр представлений $\Xi_{r, k}^{\alpha}$.

ПрЕДЛОЖЕНИЕ 3.8. Любое подпредставление в керн-представлении $T_{\alpha}$ содержит ненулевой $\mathrm{O}(p) \times \mathrm{O}(q)$-инвариантный вектор.

ДокАЗАтЕльство. Функция $f(z)=1$ является О $(p, q)$-циклическим вектором в $H_{\alpha}^{\circ}$ (так как ее орбита в точности состоит из всех векторов переполненного базиса). Поэтому проекция функции $f(z)=1$ на любое инвариантное подпространство является циклическим вектором, а следовательно, отлична от нуля.

ПрЕДЛОЖЕНИЕ 3.9. (а) Представление группьл $\mathrm{O}(p, q)$ в $\Xi_{0,0}^{\alpha}$ неприводимо.

(b) Представление группьл $\mathrm{O}(p, q)$ в $\Xi_{0, k}^{\alpha}$ есть конечная сумма неприводимьх представлений.

ДокАЗАтельство. (а) Действительно на $\mathrm{M}_{0}$ есть ровно одна $\mathrm{O}(p) \times \mathrm{O}(q)$-инвариантная функция, а именно константа. 
(b) Достаточно убедиться в том, что пространство $\mathrm{O}(p) \times \mathrm{O}(q)$-инвариантных сечений расслоения $\mathrm{S}^{k} \mathscr{N}\left(\mathrm{M}_{0}\right)$ конечномерно. Но размерность этого пространства заведомо не превосходит размерности слоя.

ЗАмечАниЕ. Блок $\Xi_{0,0}^{\alpha}$, соответствующий оператору ограничения на компактную орбиту $\mathrm{M}_{0}$, был обнаружен в работе Г. И. Ольшанского и автора $[20, \S 3]$ при более жестких ограничениях на параметры представления $(\alpha<$ $(q-2 p+1) / 2)$.

ЗАмечАниЕ. Можно показать, что спектр, соответствующий блокам $\Xi_{r, k}^{\alpha}$ при $0<r<p$, является чисто непрерывным. Можно также показать, что он состоит из представлений, которые получаются унитарным индуцированием с представлений группы $\mathrm{O}(p-r, q-r)$, лежащих в спектре представления $\Xi_{0, k}^{\alpha+2 k}(p-r, q-r)$. Эти утверждения доказываются с помощью формулы Планшереля, полученной в [19]. Работа [27] содержит полное описание спектра обсуждаемого представления.

ЗАмечАниЕ. «Добавочный» кусок непрерывного спектра в керн-представлениях ранее был обнаружен лишь в случае группы $\mathrm{U}(2,2)$ в работе [23]. B [18] была высказана гипотеза о том, что спектр керн-представления при малых значениях параметра $\alpha$ распадается на большое число разнотипных кусков (собственно, эта гипотеза и доказывается в настоящей статье).

\section{$\S 4$. Общие керн-представления}

4.1. Определение. Через $\rho_{\Lambda}$ мы обозначим конечномерное голоморфное представление группы $\mathrm{GL}(n, \mathbb{C})$, определяемое сигнатурой $\Lambda=\left(\lambda_{1}, \ldots, \lambda_{n}\right)$, где $\lambda_{1} \geqslant \cdots \geqslant \lambda_{n}$, а через $V_{\Lambda}$ - его пространство представления. Напомним, что в $V_{\Lambda}$ есть каноническое $\mathrm{U}(n)$-инвариантное скалярное произведение.

Рассмотрим представления $\rho_{\Lambda^{\prime}}, \rho_{\Lambda^{\prime \prime}}$ групп $\operatorname{GL}(p, \mathbb{C}), \operatorname{GL}(q, \mathbb{C})$, определяемые сигнатурами $\Lambda^{\prime}=\left(\lambda_{1}^{\prime}, \ldots, \lambda_{p}^{\prime}\right), \Lambda^{\prime \prime}=\left(\lambda_{1}^{\prime \prime}, \ldots, \lambda_{q}^{\prime \prime}\right)$ соответственно. Введем обозначение $\widetilde{\Lambda}=\left(\lambda_{1}^{\prime}, \ldots, \lambda_{p}^{\prime} ; \lambda_{1}^{\prime \prime}, \ldots, \lambda_{q}^{\prime \prime}\right)$, а также $V_{\widetilde{\Lambda}}=V_{\Lambda^{\prime}} \otimes V_{\Lambda^{\prime \prime}}$.

Далее, рассмотрим функцию

$$
K_{\widetilde{\Lambda}}(z, u)=\rho_{\Lambda^{\prime}}\left(1-z u^{t}\right) \otimes \rho_{\Lambda^{\prime \prime}}\left(1-u z^{t}\right), \quad z, u \in \mathrm{B}_{p, q},
$$

принимающую значения в пространстве $\operatorname{End}\left(V_{\widetilde{\Lambda}}\right)$ операторов в $V_{\widetilde{\Lambda}}$.

Допустим, что $K_{\widetilde{\Lambda}}(z, u)$ является матричнозначным положительно определенным ядром. Напомним, что это означает положительную определенность ядра

$$
L((z, \xi) ;(u, \eta))=\left\langle K_{\widetilde{\Lambda}}(z, u) \xi, \eta\right\rangle_{V_{\widetilde{\Lambda}}}, \quad x, y \in \mathrm{B}_{p, q}, \xi, \eta \in V_{\widetilde{\Lambda}},
$$

на пространстве $\mathscr{X}=\mathrm{B}_{p, q} \times V_{\widetilde{\Lambda}}$ (условие положительной определенности ядра $K_{\widetilde{\Lambda}}(z, u)$ см., например, в $\left.[20]\right)$.

Ядро $L((z, \xi) ;(u, \eta))$ определяет некоторое гильбертово пространство, состоящее из функций на $\mathscr{X}=\mathrm{B}_{p, q} \times V_{\widetilde{\Lambda}}$. Но эти функции линейны по переменной $\xi \in V_{\widetilde{\Lambda}}$; поэтому их можно рассматривать как $V_{\widetilde{\Lambda}}$-значные функции на $\mathrm{B}_{p, q}$.

В итоге мы получаем некоторое пространство $H_{\widetilde{\Lambda}}^{\circ}$, состоящее из $V_{\widetilde{\Lambda}}$-значных функций на $\mathrm{B}_{p, q}$. Группа $\mathrm{O}(p, q)$ действует в $H_{\widetilde{\Lambda}}^{\circ}$ унитарными операторами по формуле

$$
T_{\widetilde{\Lambda}}(g) f(z)=\left[\rho_{\Lambda^{\prime}}(a+z c) \otimes \rho_{\Lambda^{\prime \prime}}\left(-d+c z^{[g]}\right)\right] f\left(z^{[g]}\right) .
$$




\section{2. Операторы ограничения на границу.}

Tеорема 4.1. (а) Пусть $\lambda_{1}^{\prime \prime}-\lambda_{p}^{\prime}<(q-p+2 r) / 2$. Тогда в $H_{\widetilde{\Lambda}}^{\circ}$ корректно определен оператор ограничения функиии на $\mathrm{M}_{r}$.

(b) Пусть $2 k+\lambda_{1}^{\prime \prime}-\lambda_{p}^{\prime}<(q-p+2 r) / 2$. Тогда в $H_{\widetilde{\Lambda}}^{\circ}$ корректно определен оператор ограничения $k$-х частных производных функции на $\mathrm{M}_{r}$.

ДоКАЗАТЕЛЬСТвО аналогично доказательству теоремы 3.2 , но нужно дополнительно использовать несложные соображения из [20, 4.5].

4.3. Комментарии. Так же, как и в предыдущем параграфе, мы получаем разбиение керн-представления на блоки $\Xi_{r, k}^{\Lambda}$, где $r$ - номер орбиты, а $k-$ порядок производной.

Существование оператора ограничения на $\mathrm{M}_{0}$ было обнаружено в $[20, \S 4]$ (при более жестких ограничениях на сигнатуру $\Lambda$ ). Это, в частности, дает простой способ строить некоторые «экзотические» унитарные представления групп $\mathrm{O}(p, q)$ (см. $[20, \S \S 4-5])$. Отметим также, что эти представления выдерживают предельный переход при $q \rightarrow \infty[20, \S 6]$.

Представления группы $\mathrm{O}(p, q)$ в $\Xi_{0,0}^{\Lambda}$ являются приводимыми, их разложение обсуждается в $[20, \S 5]$. По-прежнему представление группы $\mathrm{O}(p, q)$ во всех пространствах $\Xi_{0, k}^{\Lambda}$ имеет чисто дискретный спектр. Однако спектр представления группы $\mathrm{O}(p, q)$ в блоках $\Xi_{r, k}^{\Lambda}$ при $r>0$ сам по себе устроен весьма замысловато и распадается на разнотипные куски. У меня нет правдоподобных предположений о его структуре.

\section{ЛИТЕРАТУРА}

1. Березин Ф. А. Квантование в комплексных симметрических областях. Изв. АН СССР, сер. матем., 39, № 2, 1362-1402 (1975).

2. Березин $\Phi$. А. О связи между ко- и контравариантными символами операторов в классических комплексных областях. ДАН СССР, 241, № 1, 15-17 (1978).

3. van Dijk H., Hille S. C. Canonical representations related to hyperbolic spaces. J. Funct. Anal., 147, 109-139 (1997).

4. van Dijk G., Molchanov V.F. The Berezin form for rank 1 para-hermitian symmetric spaces. J. Math. Pure Appl., 77, 747-799 (1998).

5. Faraut J., Koranyi A. Analysis in symmetric cones. Oxford Univ. Press, 1994.

6. Faraut J., Olafsson G. Causal semisimple symmetric spaces, the geometry and harmonic analysis. In: Semigroups in Agebra, Geometry and Analysis (Hofmann K. H., Lawson J. D., Vinberg E. B., eds.). Walter de Gruyter, Berlin, 1995, pp. 3-32.

7. Гантмахер Ф. Р. Теория матриц. 4-е изд., Наука, М., 1988.

8. Гельфанд И. М., Гиндикин С. Г. Комплексные многообразия, чьи остовы являются полупростыми вещественными группами Ли, и голоморфные дискретные серии представлений. Функц. анализ и его прил., 11, вып. 4, 19-27 (1977).

9. Gindikin S. Conformal analysis on hyperboloids. J. Geom. Phys., 10, 175-184 (1993).

10. Gutkin E. Coeffitients of Clebsch-Gordan for holomorphic discrete series. Lett. Math. Phys., 3, 185-192 (1979).

11. Хуа Ло Кен. Гармонический анализ функций многих комплексных переменных в классических областях. ИЛ, М., 1959.

12. Jacobsen H., Vergne $M$. Restrictions and expansions of holomorphic representations. J. Funct. Anal., 34, 29-53 (1979).

13. Лившии M. C. О спектральном разложении линейных несамосопряженных операторов. Матем. сб., 34, 145-199 (1954). 
14. Молчанов В. Ф. Разделение серий для гиперболоидов. Функц. анализ и его прил., 31, вып. 3, 35-43 (1997).

15. Неретин Ю. А. О дискретных вхождениях представлений дополнительной серии в тензорные произведения унитарных представлений. Функц. анализ и его прил., 20, вып. 4, 79-80 (1986).

16. Неретин Ю. А. Категории симметрий и конечномерные группы. УРСС, М., 1998.

17. Неретин Ю. А. Ограничение функции, голоморфной в области, на кривую, лежащую в границе, и дискретные $\mathrm{SL}_{2}(\mathbb{R})$-спектры. Изв. РАН, сер. матем., 62, № 3, 67-86 (1998).

18. Neretin $Y u$. A. Boundary values of holomorphic functions and some spectral problrms for unitary representations. In: Positivity in Lie Groups: Open Problems (Hilgert J., Lawson J. D., Need K.-H., Vinberg E. B., eds.), Walter de Gruyter, Berlin, 1998.

19. Neretin $Y u$. A. Matrix analogues of $B$-function and Plancherel formula for Berezin kernel-representations. Preprint, http://xxx.itep.ru/abs/math/9905045.

20. Неретин Ю. А., Ольшанский Г. И. Граничные значения голоморфных функций, особые унитарные представления групп $\mathrm{O}(p, q)$ и их пределы при $q \rightarrow \infty$. Зап. научн. семин. ПОМИ РАН, 223, 9-91 (1995); English transl.: J. Math. Sci., 87, No. 6, 3983-4035 (1997).

21. Ólafsson G., Orsted B. Bargmann transform for symmetric spaces. In: Lie Theory and Its Applications in Physics (Doebner H., Dobrev V. K., Hilgert J., eds.), World Scientific, 1996, pp. 3-15.

22. Ольшанский Г. И. Комплексные полугруппы Ли, пространства Харди и программа Гельфанда-Гиндикина. В сб.: Вопросы теории групп и гомологической алгебры. Изд-во Ярославского гос. университета, 1982, с. 85-98; English transl.: Differential Geometry and Its Applications, 1, No. 1, 297-308 (1991).

23. Orsted B., Zhang G. Tensor products of analytic continuations of discrete series. Can. J. Math., 49, No. 6, 1224-1241 (1997).

24. Repka J. On tensor products of holomorphic discrete series. Can. J. Math., 31, 638-844 (1979).

25. Unterberger A., Upmeier $H$. The Berezin transform and invariant differential operators. Comm. Math. Phys., 164, 563-597 (1994).

26. Вершик А. М., Гельфанд И. М., Граев M. И. Представления $\mathrm{SL}_{2}(R)$, где $R-$ кольцо функций. УМН, 28, вып. 3, 3-41 (1973).

27. Neretin $Y u$. A. Plancherel formula for Berezin deformations of $L^{2}$ on Riemannian symmetric space. Preprint, http://xxx.itep.ru/math/9911020.

Московский государственный институт электроники и математики

Поступило в редакцию 24 ноября 1998 г. 